\title{
Two- and Three-Pion Finite-Volume Spectra at Maximal Isospin from Lattice QCD
}

\author{
Ben Hörz『* \\ Nuclear Science Division, Lawrence Berkeley National Laboratory, Berkeley, California 94720, USA \\ Andrew Hanlon ${ }^{\dagger}$ \\ Helmholtz-Institut Mainz, Johannes Gutenberg-Universität, 55099 Mainz, Germany
}

(Received 15 May 2019; revised manuscript received 31 July 2019; published 4 October 2019)

\begin{abstract}
We present the three-pion spectrum with maximal isospin in a finite volume determined from lattice QCD, including excited states in addition to the ground states across various irreducible representations at zero and nonzero total momentum. The required correlation functions, from which the spectrum is extracted, are computed using a newly implemented algorithm which speeds up the computation by more than an order of magnitude. On a subset of the data we extract a nonzero value of the three-pion threshold scattering amplitude using the $1 / L$ expansion of the three-particle quantization condition, which consistently describes all states at zero total momentum. The finite-volume spectrum is publicly available to facilitate further explorations within the available three-particle finite-volume approaches.
\end{abstract}

DOI: 10.1103/PhysRevLett.123.142002

Introduction.-Lattice QCD calculations of scattering amplitudes have matured significantly over the last decade owing to marked increases in available computational capacity and improved algorithms. A widely used approach for constraining scattering observables from simulations relies on precise measurements of the interacting energy levels of QCD in a finite volume, which encode hadron interactions via the shifts from their noninteracting values [1-5] (see Ref. [6] for a survey of extensions of the formalism and numerical results).

So far, practical calculations in lattice QCD have been mostly confined to the two-hadron sector. Though a large abundance of lattice data is currently available for scattering of two hadrons (e.g., $\pi \pi$ scattering in all three isospin channels [7-31], see also Refs. [32,33] for results using a potential-based approach), these calculations are formally restricted to energies below thresholds involving three or more hadrons due to the use of a formalism for relating finite-volume spectra to scattering amplitudes that is limited to two-hadron scattering. This limitation has precluded a proper lattice QCD study of systems involving three or more stable hadrons at light pion masses, e.g., the Roper resonance which decays to both two- and threeparticle channels, the $\omega(782)$ decaying to three pions, many of the $X, Y$, and $Z$ resonances, and three-nucleon interactions relevant for nuclear physics.

Published by the American Physical Society under the terms of the Creative Commons Attribution 4.0 International license. Further distribution of this work must maintain attribution to the author(s) and the published article's title, journal citation, and DOI. Funded by SCOAP .
Following the demonstration that the finite-volume spectrum is determined by the infinite-volume $S$ matrix, even in the presence of three-particle intermediate states [34], significant progress has been made in developing the necessary formalism to interpret the three-particle finitevolume spectrum, both by extending the two-particle derivation to include three-hadron states [35-38], as well as through alternative approaches [39-43] (for a review see Ref. [44]) [45]. Thus, although the three-particle formalism is quite mature-including numerical explorations of the corresponding quantization conditions [49-51]—data for three-particle finite-volume QCD spectra are lacking, since previous lattice QCD calculations have been restricted to the extraction of multimeson ground states at rest [52-54]. Recently, a first calculation of finite-volume spectra including three-particle energies was carried out in the $b_{1}$ system, whose results are however not yet amenable to an interpretation in the present three-particle formalisms [55]. Hence no comprehensive data exist to apply the available finite-volume formalisms.

We fill this gap by providing the two-pion and three-pion spectra with maximum isospin in various irreducible representations (irreps) at zero and nonzero total momentum in the elastic region, i.e., for center-of-mass energies $E_{\mathrm{cm}} / m_{\pi}$ below 4 and 5 for isospin $I=2$ and $I=3$, respectively. Our analysis of a subset of the data indicates sensitivity to the three-pion interaction at the current level of precision. In order to facilitate a more detailed exploration, possibly including the effect of higher partial waves $[35,36,43,56]$, the spectrum data are made public, including all correlations.

A technical challenge concerns the growing number of Wick contractions required to compute correlation 
functions of suitable interpolating operators-from which the spectrum is extracted-as the number of valence quark fields increases. The continued need for improved algorithms to perform these contractions was pointed out recently [57] and indeed was a limiting factor in a recent study of meson-baryon scattering in the $\Delta$ channel [58]. While Refs. [59-64] investigated efficient contraction algorithms at the quark level, we employ the stochastic variant [65] of distillation [66] to treat quark propagation. In this framework, it is useful to view the correlation function construction in terms of contractions of tensors associated with the involved hadrons. Then, to reduce the operation count required to evaluate all contractions, we use a method which is well-known in quantum chemistry [67-69] and has attracted renewed interest in the context of tensor networks [70]. The proposed optimization achieves a speed up by more than an order of magnitude, can be readily used for general physical systems (e.g., three-meson systems at nonmaximal isospin and two-baryon systems), and its implementation is publicly available [71].

This Letter is organized as follows: We first discuss the interpolating operators employed and construction of their correlation functions, followed by a description of the ensemble used in this work. Subsequently, the finite-volume spectra and extraction of two- and threepion scattering parameters from a subset of the data are presented.

Interpolating operators: Lattice simulations of QCD in a cubic box break the infinite-volume $S O(3)$ rotational symmetry. The spectrum in finite volume is customarily extracted employing interpolating operators which transform irreducibly under the symmetries of a cubic spatial lattice, i.e., the octahedral group $O_{h}$ for zero total momentum $\mathbf{P}=\mathbf{0}$ and the corresponding little groups for $\mathbf{P} \neq \mathbf{0}$ $[15,72]$. Correlation functions of such interpolators access only the sub-block of the finite-volume Hamiltonian corresponding to the same irrep, thus greatly simplifying the determination of the spectrum and the subsequent scattering-amplitude analysis.

We employ the simplest single-pion operator destroying a three-momentum $\mathbf{p}$ given by

$$
\pi_{\mathbf{p}}(t)=\sum_{\mathbf{x}} e^{-i \mathbf{p} \cdot \mathbf{x}} \bar{d}(\mathbf{x}, t) \gamma_{5} u(\mathbf{x}, t) .
$$

This operator transforms in the $A_{1 u}^{-}$and $A_{2}^{-}$irrep for zero and nonzero momentum, respectively, where the superscript specifies the $G$ parity.

Two-pion interpolators which transform according to the irrep $\Lambda$ of the little group of total momentum $\mathbf{P}$ are obtained by forming appropriate linear combinations of two singlepion interpolators with momenta $\mathbf{p}_{1}+\mathbf{p}_{2}=\mathbf{P}$,

$$
\pi \pi^{(\mathbf{P}, \Lambda)}(t)=c_{\mathbf{p}_{1}, \mathbf{p}_{2}}^{(\mathbf{P}, \Lambda)} \pi_{\mathbf{p}_{1}}(t) \pi_{\mathbf{p}_{2}}(t) .
$$

The relevant Clebsch-Gordan coefficients $c_{\mathbf{p}_{1}, \mathbf{p}_{2}}^{(\mathbf{P}, \Lambda)}$ were worked out in Ref. [72] (see also Ref. [15]) and used previously to study $\pi \pi$ scattering $[22,29]$.

Three-pion interpolators are obtained by iterating this process, i.e., by first coupling two of the pions into an intermediate irrep, then using the Clebsch-Gordan coefficients again to obtain operators transforming according to one of the total irreps of interest,

$$
\pi \pi \pi^{(\mathbf{P}, \Lambda)}(t)=c_{\mathbf{p}_{1}, \mathbf{p}_{2}, \mathbf{p}_{3}}^{\left(\mathbf{P}, \pi_{\mathbf{p}_{1}}\right.}(t) \pi_{\mathbf{p}_{2}}(t) \pi_{\mathbf{p}_{3}}(t)
$$

Because of the weak interaction in $I=2 \pi \pi$ scattering, which is the only relevant subprocess for this work, the more elaborate operator construction discussed in Ref. [55] is not required. The interpolators we use in this work are listed in the Supplemental Material [73].

Correlation function construction: Quark propagation is treated using the stochastic LapH method [65] by first obtaining smeared solutions of the Dirac equation,

$$
\varphi^{[r, d]}=\mathcal{S} D^{-1} \varrho^{[r, d]},
$$

for stochastic quark-field sources $Q^{[r, d]}$ with noise index $r=1, \ldots, N_{\eta}$, dilution [74,75] index $d=1, \ldots, N_{\mathrm{dil}}$, and where $\mathcal{S}$ is the LapH smearing kernel, formed from the $N_{\mathrm{ev}}$ lowest eigenvectors of the three-dimensional covariant Laplacian. Next, useful intermediate quantities are the pion source and sink functions [65]

$$
\begin{aligned}
& \mathcal{M}_{\mathbf{p}}^{\left[r_{1}, r_{2}, d_{1}, d_{2}\right]}(t)=-\sum_{\mathbf{x}} e^{-i \mathbf{p} \cdot \mathbf{x}} \varrho_{a \alpha \mathbf{x} t}^{\left[r_{1}, d_{1}\right] *} \varrho_{a \alpha \mathbf{x} t}^{\left[r_{2}, d_{2}\right]}, \\
& \overline{\mathcal{M}}_{\mathbf{p}}^{\left[r_{1}, r_{2}, d_{1}, d_{2}\right]}(t)=\sum_{\mathbf{x}} e^{-i \mathbf{p} \cdot \mathbf{x}} \varphi_{a \alpha \mathbf{x} t}^{\left[r_{1}, d_{1}\right] *} \varphi_{a \alpha \mathbf{x} t}^{\left[r_{2}, d_{2}\right]},
\end{aligned}
$$

with summed color index $a$ and spin index $\alpha$, and two open noise and dilution indices. In terms of these meson functions, the single-pion correlation function on a single gauge configuration is obtained by the average over noise combinations $\left\{r_{1}, r_{2}\right\}$ [65],

$C_{\pi_{\mathbf{p}}}\left(t_{f}-t_{s}\right) \propto-\sum_{\left\{r_{1}, r_{2}\right\}} \overline{\mathcal{M}}_{\mathbf{p}}^{\left[r_{1}, r_{2}, d_{1}, d_{2}\right]}\left(t_{f}\right) \mathcal{M}_{\mathbf{p}}^{\left[r_{1}, r_{2}, d_{1}, d_{2}\right] *}\left(t_{s}\right)$,

with proper normalization given by the number of noise combinations used to perform the average.

For a given momentum, pair of source and sink time $t_{s}$ and $t_{f}$, and noise combination, Eq. (6) is a tensor contraction over dilution indices of two rank-2 tensors with index range $N_{\text {dil }}$. Two- and three-pion correlation functions with maximal isospin can be computed using the same building blocks [65] and involve tensor contractions governed by all possible Wick contractions of four and six rank-2 tensors, respectively. 


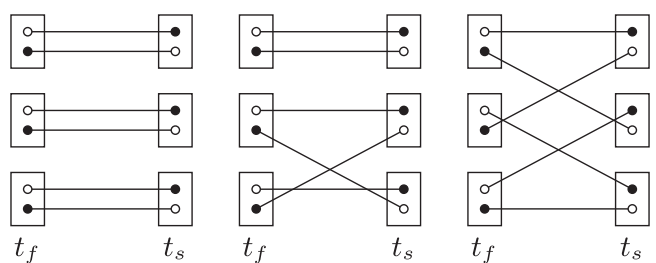

FIG. 1. Different topologies of Wick contractions required to evaluate $I=3$ three-pion correlation functions. Circles indicate quark and antiquark fields tied into meson functions shown as boxes, which are subsequently contracted. Two-pion $I=2$ Wick contractions appear as subexpressions.

The number of Wick contractions grows factorially as more pions are included [52], and the different topologies of diagrams for three-pion correlation functions in the sector of maximal isospin are shown in Fig. 1. However, across the relevant diagrams there is a lot of redundancy which can be exploited systematically to reduce the number of arithmetic operations required for their evaluation. In particular, all diagrams required for the computation of $I=$ 2 two-pion correlation functions appear as subdiagrams of $I=3$ three-pion correlation functions.

The algorithm to automatically perform the operationcount minimization is described in the Supplemental Material [73] together with a detailed example. For the evaluation of the correlation functions required in this work we achieve a speed up by roughly a factor of 15 .

Ensemble details: The results in this work are based on the D200 ensemble generated through the CLS effort [76] with $N_{\mathrm{f}}=2+1$ quark flavors at a pion mass $m_{\pi} \approx$ $200 \mathrm{MeV}$ and lattice spacing $a \approx 0.064 \mathrm{fm}$ [77]. The ensemble and measurement setup is detailed in Table I. In order to ensure a Hermitian matrix of correlation functions despite the use of open boundary conditions in the temporal direction [78], our interpolating operators are always separated from the temporal boundaries by at least $t_{s}$, where $m_{\pi} t_{s}=2.2$.

Pion-pion scattering in the isovector channel has been investigated on this ensemble previously [29], and statistics subsequently improved considerably to provide spectroscopic information for the determination of the hadronic vacuum polarization on the same ensemble [80]. The pion

TABLE I. Ensemble and measurement setup used in this work. Measurements are performed on $N_{\text {cfg }}$ configurations with spatial volume $L^{3}$ separated by 4 MDU. Correlation functions are estimated starting from a single source time $t_{s} / a=35$ using $N_{\eta}$ diluted noise sources to estimate quark propagators (see Ref. [65] for unexplained notation). Other parameters, e.g., for stout smearing [79], are the same as in Ref. [29].

\begin{tabular}{lccccc}
\hline \hline$a m_{\pi}$ & $L / a$ & $N_{\mathrm{ev}}$ & Dilution & $N_{\eta}$ & $N_{\mathrm{cfg}}$ \\
\hline $0.06504(33)$ & 64 & 448 & (TF,SF,LI16) & 6 & 1100 \\
\hline \hline
\end{tabular}

functions are re-used from that work, and hence no additional meson functions or solutions of the Dirac equation have to be computed.

Analysis strategy: The procedure to extract the finitevolume spectrum from a matrix of correlation functions $C_{i j}(t)$ in a given irrep is discussed in detail in Ref. [22] and we use the analysis suite [81] developed in Ref. [29].

We solve a generalized eigenvalue problem $[4,82,83]$ for a fixed reference time and diagonalization time $\left(t_{0}=5 a, t_{*}=10 a\right)$, corresponding to roughly 0.32 and $0.64 \mathrm{fm}$ in physical units [77], in order to extract not only the ground state but also excited states in most irreps. Results from different $\left(t_{0}, t_{*}\right)$ are indistinguishable, presumably due to the weak interaction in $I=2$ and $I=3$ pion scattering which results in little mixing of our interpolating operators, in which each hadron has been projected to definite momentum and is hence expected to overlap predominantly with a single state.

For two-pion states the difference $\Delta E$ between interacting and noninteracting energies is determined from singleexponential fits at sufficiently large time separations to the ratios,

$$
R_{i}(t)=\frac{\hat{C}_{i i}(t)}{C_{\pi_{\mathbf{p}_{1}}}(t) C_{\pi_{\mathbf{p}_{2}}}(t)} \stackrel{\text { large } t}{\longrightarrow} A \mathrm{e}^{-\Delta E_{i} t},
$$

of diagonal elements of the "optimized" correlation matrix $\hat{C}[22]$ and two single-pion correlation functions, and similarly for the three-pion states [52]. Absolute energies are reconstructed from those energy differences using the single-pion dispersion relation. The attainable precision is generally at the few-permille level for the energies measured in units of the single-pion mass.

Two-pion spectrum and scattering amplitude: The twopion spectrum with maximal isospin is shown in Fig. 2 together with the noninteracting energies. The respective

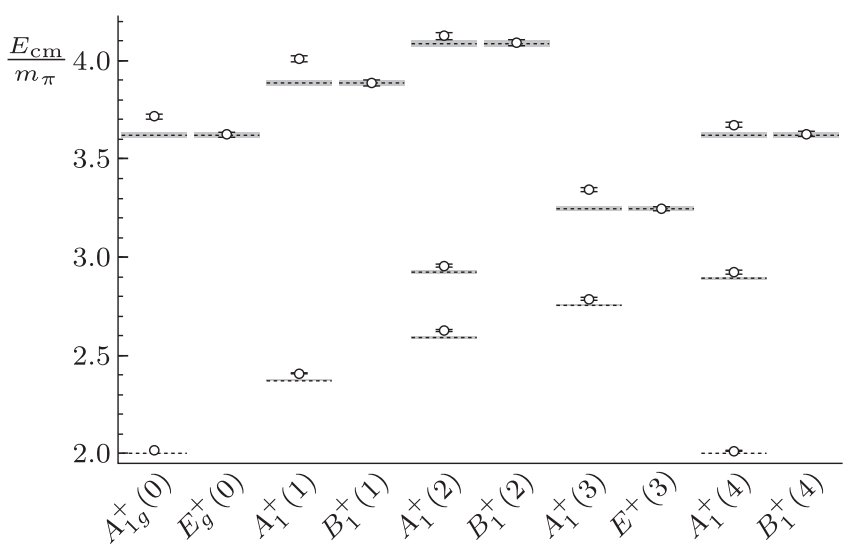

FIG. 2. $I=2$ two-pion spectrum in various irreps $\Lambda\left(\mathbf{d}^{2}\right)$ with total momentum $\mathbf{P}=(2 \pi / L)$ d. Open symbols denote the measured interacting energies which are shifted from their noninteracting values shown as dashed lines. 


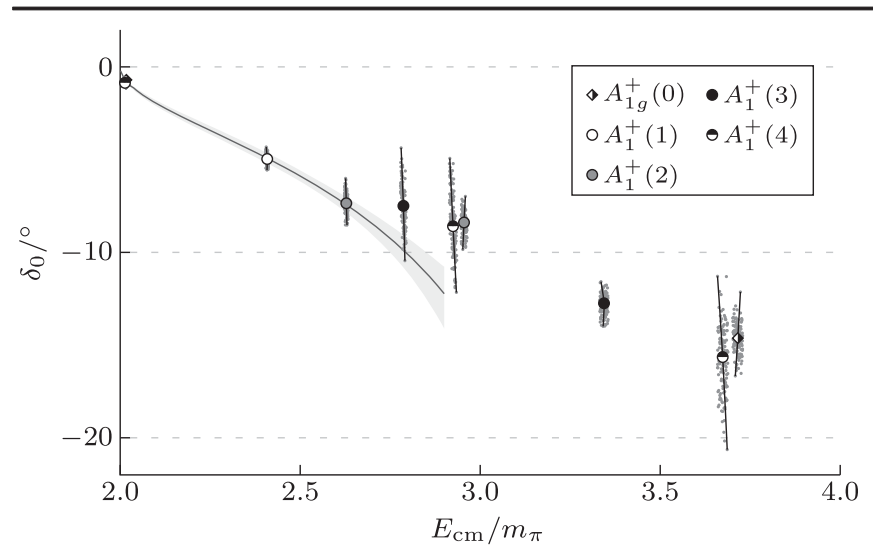

FIG. 3. Energy dependence of the $I=2 \pi \pi s$-wave scattering amplitude extracted from the two-pion spectrum. The gray band shows the result of the fit to the five leftmost points using the effective range expansion given in Eq. (8).

differences encode the two-pion scattering amplitude for $\ell=0$ shown in Fig. 3 neglecting the effect of higher even partial waves. Its energy dependence for scattering momentum $q^{2} / m_{\pi}^{2}<1$ is described using the effectiverange expansion

$$
q \cot \delta_{0}=-\frac{1}{a_{0}}+\frac{r_{0}}{2} q^{2},
$$

and the scattering length $a_{0}$ and effective range $r_{0}$ are determined from a fit using the determinant-residual method [84] with $\mu=64$, which yields

$$
\begin{aligned}
m_{\pi} a_{0} & =0.1019(88), \quad m_{\pi} r_{0}=9.0(2.4), \\
\chi^{2} / \text { dof } & =1.33 .
\end{aligned}
$$

A comparison of our scattering length with previous lattice QCD determinations [7-9,15,17,18,22], together with the experimental value from Ref. [85], is shown in the Supplemental Material [73].

Three-pion spectrum and $1 / L$ expansion: The threepion spectrum with maximal isospin is shown in Fig. 4, displaying significant energy shifts in all irreps. In particular, interacting energy levels from different irreps that contain some degeneracy of the noninteracting spectra (e.g., $A_{1 u}^{-}$and $E_{u}^{-}$at zero total momentum) differ substantially, which may suggest sensitivity to different combinations of low-energy scattering parameters.

At leading order in the $1 / L$ expansion of the threeparticle quantization condition [86-90] [91],

$$
\begin{aligned}
\Delta E_{3}= & \frac{12 \pi a_{0}}{m_{\pi} L^{3}}\left\{1-\left(\frac{a_{0}}{\pi L}\right) \mathcal{I}+\left(\frac{a_{0}}{\pi L}\right)^{2}\left(\mathcal{I}^{2}+\mathcal{J}\right)+\frac{3 \pi a_{0}}{m_{\pi}^{2} L^{3}}\right. \\
& +\frac{64 \pi^{2} a_{0}^{2} \mathcal{C}_{3}}{m_{\pi} L^{3}}+\frac{6 \pi r_{0} a_{0}^{2}}{L^{3}}+\left(\frac{a_{0}}{\pi L}\right)^{3}\left[c_{L} \log \left(N_{\mathrm{cut}}\right)-\mathcal{I}^{3}+\mathcal{I} \mathcal{J}\right. \\
& \left.\left.+15 \mathcal{K}+\mathcal{C}_{F}+\mathcal{C}_{4}+\mathcal{C}_{5}\right]\right\}-\frac{\mathcal{M}_{3, \text { th }}}{48 m_{\pi}^{3} L^{6}}+O\left(L^{-7}\right)
\end{aligned}
$$

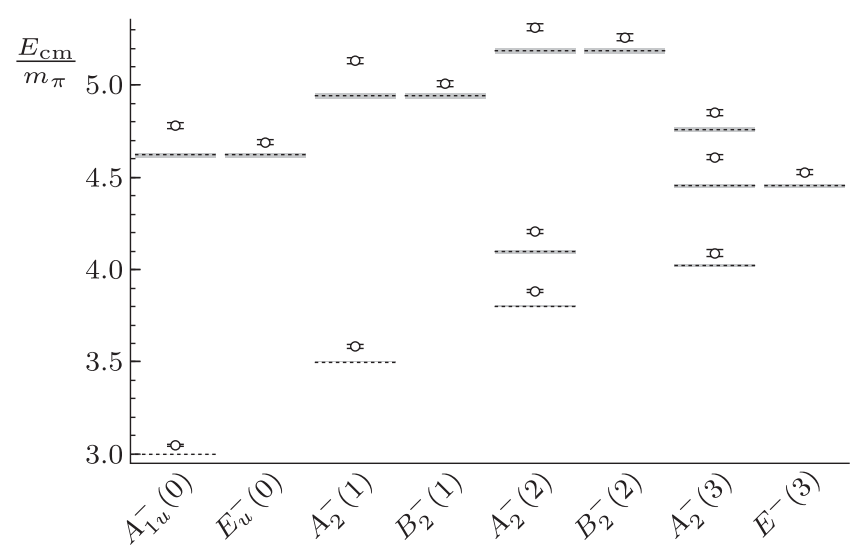

FIG. 4. Same as Fig. 2 but for the $I=3$ three-pion spectrum.

the ground-state energy shift $\Delta E_{3}$ is 3 times larger than the corresponding two-particle shift $\Delta E_{2}$ [52]. The deviation of our numerical result

$$
\Delta E_{3} / \Delta E_{2}=2.78(21)
$$

is due to two-particle effects at higher orders in $1 / L$ and the three-pion interaction. Using the two-particle scattering length and effective range determined before, the threeparticle threshold scattering amplitude entering at $L^{-6}$ can be isolated and we obtain

$$
\frac{m_{\pi}^{2} \mathcal{M}_{3, \mathrm{th}}}{48\left(m_{\pi} L\right)^{6}}=0.0113(43)
$$

While this quantity depends on how two- and three-particle effects are separated $[50,88]$, within the scheme discussed in those references our result indicates sensitivity to threeparticle physics.

Using the nonrelativistic threshold expansion from Ref. [90] yields a result with similar significance. From that reference, the energy of the excited states at rest are predicted to be

$$
\begin{gathered}
A_{1 u}^{-}: \quad E_{3}^{\prime} / m_{\pi}=4.76(8), \\
E_{u}^{-}: \quad E_{3}^{\prime} / m_{\pi}=4.72(2),
\end{gathered}
$$

in good agreement with our measured values. Further, the formalism employed in Ref. [51] with the scattering parameters determined there predicts the first excited state in the at-rest $A_{1 u}^{-}$irrep to be [92]

$$
E_{3}^{\prime} / m_{\pi}=4.75
$$

at our pion mass and spatial volume, which is also in agreement with the measured value.

More work along the lines of Refs. $[43,56]$ is required to apply the quantization condition to the energies in all irreps presented here. In order to facilitate further investigations in that direction, the two-pion and three-pion spectra are made 
publicly available including all correlations. The values and covariance matrix of all extracted energies, as well as the single-pion mass, are given in the Supplemental Material [73], and the bootstrap samples from this analysis are available as ancillary files with the arXiv submission.

Conclusions and outlook.-We have presented the $I=3$ three-pion spectrum in finite volume from lattice QCD in which, for the first time, the excited states in various irreps at zero and nonzero total momentum have been extracted. The nonrelativistic three-particle $1 / L$ expansion consistently describes the levels in the irreps at zero total momentum. However, the entire spectrum should be interpreted in the framework of a full three-particle finite-volume formalism in order to corroborate and extend our extraction of the three-pion interaction. In the interest of facilitating those investigations, which will require generalizations of the formulas currently available in the literature, all spectra are made public, including their correlations.

We also described a method to reduce the computational cost of constructing correlation functions in the stochastic variant of distillation, achieving a speed up of more than an order of magnitude for the set of observables considered here. This algorithmic improvement paves the way to study more complicated systems such as the Roper resonance, which has a sizable branching ratio for decays to $N \pi \pi$ as well as $N \pi$, and also reduces the computational cost associated with correlation function construction for baryon-baryon systems, which will facilitate the lattice QCD investigation of nucleon-nucleon as well as nucleonhyperon interactions relevant for nuclear physics.

We acknowledge helpful discussions with Andria Agadjanov, John Bulava, Ken McElvain, Daniel Mohler, Colin Morningstar, Fernando Romero-López, Akaki Rusetsky, and André Walker-Loud. We thank Christopher Körber for useful comments on an earlier version of the manuscript. The authors thank the Yukawa Institute for Theoretical Physics at Kyoto University, where part of this work was performed during the YITP-19-01 workshop on "Frontiers in Lattice QCD and related topics." The work of B. H. was supported by the Laboratory Directed Research and Development Program of Lawrence Berkeley National Laboratory under U.S. Department of Energy Contract No. DE-AC02-05CH11231. Calculations for this project were performed on the HPC clusters "HIMster II" at the Helmholtz-Institut Mainz and "Mogon II" at JGU Mainz. Our programs use the deflated SAP + GCR solver from the openQCD package [78], as well as the QDP++/Chroma libraries [93]. We are grateful to our colleagues in the CLS initiative for sharing ensembles.

*hoerz@lbl.gov

†ahanlon@uni-mainz.de

[1] M. Luscher, Commun. Math. Phys. 104, 177 (1986).
[2] M. Luscher, Commun. Math. Phys. 105, 153 (1986).

[3] M. Lüscher, Nucl. Phys. B354, 531 (1991).

[4] M. Luscher and U. Wolff, Nucl. Phys. B339, 222 (1990).

[5] K. Rummukainen and S. A. Gottlieb, Nucl. Phys. B450, 397 (1995).

[6] R. A. Briceño, J. J. Dudek, and R. D. Young, Rev. Mod. Phys. 90, 025001 (2018).

[7] S. R. Beane, P. R. Bedaque, K. Orginos, and M. J. Savage (NPLQCD Collaboration), Phys. Rev. D 73, 054503 (2006).

[8] S. R. Beane, T. C. Luu, K. Orginos, A. Parreno, M. J. Savage, A. Torok, and A. Walker-Loud, Phys. Rev. D 77, 014505 (2008).

[9] X. Feng, K. Jansen, and D. B. Renner, Phys. Lett. B 684, 268 (2010).

[10] X. Feng, K. Jansen, and D. B. Renner, Phys. Rev. D 83, 094505 (2011).

[11] S. R. Beane, E. Chang, W. Detmold, H. W. Lin, T. C. Luu, K. Orginos, A. Parreno, M. J. Savage, A. Torok, and A. Walker-Loud (NPLQCD Collaboration), Phys. Rev. D 85, 034505 (2012).

[12] C. B. Lang, D. Mohler, S. Prelovsek, and M. Vidmar, Phys. Rev. D 84, 054503 (2011); 89, 059903 (2014).

[13] S. Aoki et al. (CS Collaboration), Phys. Rev. D 84, 094505 (2011).

[14] C. Pelissier and A. Alexandru, Phys. Rev. D 87, 014503 (2013).

[15] J. J. Dudek, R. G. Edwards, and C. E. Thomas, Phys. Rev. D 86, 034031 (2012).

[16] J. J. Dudek, R. G. Edwards, and C. E. Thomas (Hadron Spectrum Collaboration), Phys. Rev. D 87, 034505 (2013).

[17] K. Sasaki, N. Ishizuka, M. Oka, and T. Yamazaki (PACS-CS Collaboration), Phys. Rev. D 89, 054502 (2014).

[18] Z. Fu, Phys. Rev. D 87, 074501 (2013).

[19] D. J. Wilson, R. A. Briceño, J. J. Dudek, R. G. Edwards, and C. E. Thomas, Phys. Rev. D 92, 094502 (2015).

[20] C. Helmes, C. Jost, B. Knippschild, C. Liu, J. Liu, L. Liu, C. Urbach, M. Ueding, Z. Wang, and M. Werner (ETM Collaboration), J. High Energy Phys. 09 (2015) 109.

[21] G. S. Bali, S. Collins, A. Cox, G. Donald, M. Göckeler, C. B. Lang, and A. Schäfer (RQCD Collaboration), Phys. Rev. D 93, 054509 (2016).

[22] J. Bulava, B. Fahy, B. Hörz, K. J. Juge, C. Morningstar, and C. H. Wong, Nucl. Phys. B910, 842 (2016).

[23] D. Guo, A. Alexandru, R. Molina, and M. Doring, Phys. Rev. D 94, 034501 (2016).

[24] Z. Fu and L. Wang, Phys. Rev. D 94, 034505 (2016).

[25] R. A. Briceño, J. J. Dudek, R. G. Edwards, and D. J. Wilson, Phys. Rev. Lett. 118, 022002 (2017).

[26] L. Liu et al., Phys. Rev. D 96, 054516 (2017).

[27] C. Alexandrou, L. Leskovec, S. Meinel, J. Negele, S. Paul, M. Petschlies, A. Pochinsky, G. Rendon, and S. Syritsyn, Phys. Rev. D 96, 034525 (2017).

[28] R. A. Briceno, J. J. Dudek, R. G. Edwards, and D. J. Wilson, Phys. Rev. D 97, 054513 (2018).

[29] C. Andersen, J. Bulava, B. Hörz, and C. Morningstar, Nucl. Phys. B939, 145 (2019).

[30] D. Guo, A. Alexandru, R. Molina, M. Mai, and M. Döring, Phys. Rev. D 98, 014507 (2018).

[31] C. Culver, M. Mai, A. Alexandru, M. Doring, and F. X. Lee, Phys. Rev. D 100, 034509 (2019). 
[32] T. Kurth, N. Ishii, T. Doi, S. Aoki, and T. Hatsuda, J. High Energy Phys. 12 (2013) 015.

[33] Y. Akahoshi, S. Aoki, T. Aoyama, T. Doi, T. Miyamoto, and K. Sasaki, Prog. Theor. Exp. Phys. 2019, $083 B 02$ (2019).

[34] K. Polejaeva and A. Rusetsky, Eur. Phys. J. A 48, 67 (2012).

[35] M. T. Hansen and S. R. Sharpe, Phys. Rev. D 90, 116003 (2014).

[36] M. T. Hansen and S. R. Sharpe, Phys. Rev. D 92, 114509 (2015).

[37] R. A. Briceño, M. T. Hansen, and S. R. Sharpe, Phys. Rev. D 95, 074510 (2017).

[38] R. A. Briceño, M. T. Hansen, and S. R. Sharpe, Phys. Rev. D 99, 014516 (2019).

[39] D. Agadjanov, M. Doring, M. Mai, U.-G. Meißner, and A. Rusetsky, J. High Energy Phys. 06 (2016) 043.

[40] H.-W. Hammer, J.-Y. Pang, and A. Rusetsky, J. High Energy Phys. 09 (2017) 109.

[41] H. W. Hammer, J. Y. Pang, and A. Rusetsky, J. High Energy Phys. 10 (2017) 115.

[42] M. Mai and M. Döring, Eur. Phys. J. A 53, 240 (2017).

[43] M. Döring, H. W. Hammer, M. Mai, J. Y. Pang, A. Rusetsky, and J. Wu, Phys. Rev. D 97, 114508 (2018).

[44] M. T. Hansen and S. R. Sharpe, arXiv:1901.00483.

[45] See also Refs. [46-48] for a complementary approach for inclusive observables.

[46] S. Hashimoto, Prog. Theor. Exp. Phys. 2017, 053 B03 (2017).

[47] M. T. Hansen, H. B. Meyer, and D. Robaina, Phys. Rev. D 96, 094513 (2017).

[48] J. Bulava and M. T. Hansen, arXiv:1903.11735 [Phys. Rev. $\mathrm{D}$ (to be published)].

[49] R. A. Briceño, M. T. Hansen, and S. R. Sharpe, Phys. Rev. D 98, 014506 (2018).

[50] F. Romero-López, A. Rusetsky, and C. Urbach, Eur. Phys. J. C 78, 846 (2018).

[51] M. Mai and M. Doring, Phys. Rev. Lett. 122, 062503 (2019).

[52] S. R. Beane, W. Detmold, T. C. Luu, K. Orginos, M. J. Savage, and A. Torok, Phys. Rev. Lett. 100, 082004 (2008).

[53] W. Detmold, M. J. Savage, A. Torok, S. R. Beane, T. C. Luu, K. Orginos, and A. Parreno, Phys. Rev. D 78, 014507 (2008).

[54] W. Detmold, K. Orginos, M. J. Savage, and A. WalkerLoud, Phys. Rev. D 78, 054514 (2008).

[55] A. J. Woss, C. E. Thomas, J. J. Dudek, R. G. Edwards, and D. J. Wilson, arXiv:1904.04136 [Phys. Rev. D (to be published)].

[56] T. D. Blanton, F. Romero-López, and S. R. Sharpe, J. High Energy Phys. 03 (2019) 106.

[57] W. Detmold, R. G. Edwards, J. J. Dudek, M. Engelhardt, H.-W. Lin, S. Meinel, K. Orginos, and P. Shanahan, arXiv: 1904.09512.

[58] C. W. Andersen, J. Bulava, B. Hörz, and C. Morningstar, Phys. Rev. D 97, 014506 (2018).

[59] T. Yamazaki, Y. Kuramashi, and A. Ukawa (PACS-CS Collaboration), Phys. Rev. D 81, 111504(R) (2010).

[60] W. Detmold and M. J. Savage, Phys. Rev. D 82, 014511 (2010).

[61] T. Doi and M. G. Endres, Comput. Phys. Commun. 184, 117 (2013).
[62] W. Detmold and K. Orginos, Phys. Rev. D 87, 114512 (2013).

[63] J. Günther, B. C. Toth, and L. Varnhorst, Phys. Rev. D 87, 094513 (2013).

[64] H. Nemura, Comput. Phys. Commun. 207, 91 (2016).

[65] C. Morningstar, J. Bulava, J. Foley, K. J. Juge, D. Lenkner, M. Peardon, and C. H. Wong, Phys. Rev. D 83, 114505 (2011).

[66] M. Peardon, J. Bulava, J. Foley, C. Morningstar, J. Dudek, R. G. Edwards, B. Joo, H.-W. Lin, D. G. Richards, and K. J. Juge (Hadron Spectrum), Phys. Rev. D 80, 054506 (2009).

[67] A. Hartono, A. Sibiryakov, M. Nooijen, G. Baumgartner, D. Bernholdt, S. Hirata, C. Lam, R. Pitzer, J. Ramanujam, and P. Sadayappan, Technical Report No. OSU-CISRC-2/05TR10, Department of Computer Science and Engineering, The Ohio State University, 2005.

[68] A. Hartono, Q. Lu, X. Gao, S. Krishnamoorthy, M. Nooijen, G. Baumgartner, D. E. Bernholdt, V. Choppella, R. M. Pitzer, J. Ramanujam et al., in Computational ScienceICCS 2006, edited by V. N. Alexandrov, G. D. van Albada, P. M. A. Sloot, and J. Dongarra (Springer Berlin Heidelberg, Berlin, Heidelberg, 2006), pp. 267-275.

[69] A. Hartono, Q. Lu, T. Henretty, S. Krishnamoorthy, H. Zhang, G. Baumgartner, D. E. Bernholdt, M. Nooijen, R. Pitzer, J. Ramanujam et al., J. Phys. Chem. A 113, 12715 (2009).

[70] R. N. C. Pfeifer, J. Haegeman, and F. Verstraete, Phys. Rev. E 90, 033315 (2014).

[71] https://github.com/laphnn/contraction_optimizer.

[72] C. Morningstar, J. Bulava, B. Fahy, J. Foley, Y. C. Jhang, K. J. Juge, D. Lenkner, and C. H. Wong, Phys. Rev. D 88, 014511 (2013).

[73] See Supplemental Material at http://link.aps.org/ supplemental/10.1103/PhysRevLett.123.142002 for the list of interpolators used.

[74] W. Wilcox, in Numerical Challenges in Lattice Quantum Chromodynamics. Proceedings, Joint Interdisciplinary Workshop, Wuppertal, Germany, 1999 (Springer, Berlin, 1999), pp. 127-141.

[75] J. Foley, K. Jimmy Juge, A. O'Cais, M. Peardon, S. M. Ryan, and J.-I. Skullerud, Comput. Phys. Commun. 172, 145 (2005).

[76] M. Bruno et al., J. High Energy Phys. 02 (2015) 043.

[77] M. Bruno, T. Korzec, and S. Schaefer, Phys. Rev. D 95, 074504 (2017).

[78] M. Lüscher and S. Schaefer, J. High Energy Phys. 07 (2011) 036.

[79] C. Morningstar and M. J. Peardon, Phys. Rev. D 69, 054501 (2004).

[80] A. Gérardin, M. Cè, G. von Hippel, B. Hörz, H. B. Meyer, D. Mohler, K. Ottnad, J. Wilhelm, and H. Wittig, Phys. Rev. D 100, 014510 (2019).

[81] https://github.com/ebatz/jupan.

[82] C. Michael and I. Teasdale, Nucl. Phys. B215, 433 (1983).

[83] B. Blossier, M. Della Morte, G. von Hippel, T. Mendes, and R. Sommer, J. High Energy Phys. 04 (2009) 094.

[84] C. Morningstar, J. Bulava, B. Singha, R. Brett, J. Fallica, A. Hanlon, and B. Hörz, Nucl. Phys. B924, 477 (2017). 
[85] B. Ananthanarayan, G. Colangelo, J. Gasser, and H. Leutwyler, Phys. Rep. 353, 207 (2001).

[86] S. R. Beane, W. Detmold, and M. J. Savage, Phys. Rev. D 76, 074507 (2007).

[87] W. Detmold and M. J. Savage, Phys. Rev. D 77, 057502 (2008).

[88] M. T. Hansen and S. R. Sharpe, Phys. Rev. D 93, 096006 (2016); 96, 039901(E) (2017).
[89] S. R. Sharpe, Phys. Rev. D 96, 054515 (2017); 98, 099901 (2018).

[90] J.-Y. Pang, J.-J. Wu, H. W. Hammer, Ulf-G. Meißner, and A. Rusetsky, Phys. Rev. D 99, 074513 (2019).

[91] The numerical constants appearing in this expression are evaluated in Appendices A and C of Ref. [88].

[92] M. Mai (private communication).

[93] R. G. Edwards and B. Joo (SciDAC Collaboration), Nucl. Phys. B, Proc. Suppl. 140, 832 (2005). 